\title{
Effect of Body Mass Index on Serum CA125 Level in Females with PCOS
}

Received: 28-Jun-2020 Accepted: 30-Aug-2020 Published: 14-Dec-2020

\section{Hanan Abdulmaged Hasan, Mohammed Oda Selman, Mufeda Ali Jwad*}

${ }^{1}$ High Institute of Infertility Diagnosis and Assisted Reproductive Technologies, Al Nahrain University, Baghdad, Iraq. *mufedaalammar73@gmail.com

CA125 is a glycoprotein mucins (MUC16) tumor marker, used in screening, ovarian and endometrial malignancies and in monitoring therapy, early recurrences, and prognosis of these cancers. Hormonal induction for ovulation can elevate the risk of ovarian cancers so CA125 may be used for screening in PCOS as an unsafe group and the way of treatment may need to be changed in these patients. Polycystic Ovarian Syndrome (PCOS) is one of the most common hormonal disturbed diseases that can affect females in reproductive life. It has both metabolic and fertility impacts that can be seen in the reproductive period and post-menopausal life. To study the correlation between PCOS and CA125 level in relation to body weight in non-cancerous patients. 60 women were selected with age 18-42-year-old forty of them were diagnosed with PCOS according to Rotterdam criteria. They were divided into two groups according to BMI G1 was more than $25 \mathrm{~kg} / \mathrm{m}^{2}$ (overweight and obese) G2 was less than $25 \mathrm{~kg} / \mathrm{m}^{2}$ (normal) and the G3 was 20 healthy non-PCOS BMI less than $25 \mathrm{~kg} / \mathrm{m}^{2}$. Blood samples were taken in the early follicular phase for assessing luteinizing hormone ( $\mathrm{LH}$ ), follicular stimulating hormone (FSH) and, free testosterone (FT) hormone. Other blood samples were taken at the late follicular phase to measure CA125 levels using Enzyme-Linked Immune Sorbent Assay (ELISA). PCOS G2 were significantly higher in the concentration of CA125 than PCOS G1 ( $p<0.05)$, but there was no significant difference in CA125 level in groups when there is no significant difference in body mass index (BMI) between PCOS G1 and G2. P>0.05. The negative relation between CA125 concentration and weight. The tumor marker CA125 efficacy is decreased in obese women. Furthermore, PCOS not causes an increase in CA125 in the non-cancerous patient.

Keywords: CA125; PCOS; ELISA; LH; FSH; BMI. 


\section{Introduction}

Polycystic Ovarian Syndrome (PCOS) is among the commonest problems affecting women in the period of reproduction defined by hyperandrogenism, less frequent ovulation in enlarged ovaries including multiple follicular cysts (Park and Chun $\left.{ }^{[l}\right)$. This condition was known since 1935 named by Stien Levinthal syndrome, up to $10 \%$ of women are affected (Kabel, A. ${ }^{[2]}$ ). More than $70 \%$ of PCOS women complain of Infertility caused by a failure of ovulation (Selman, et al. $\left.{ }^{[3]}\right)$. The syndrome may also be associated with hyperinsulinemia, insulin resistance (IR), type 2 diabetes mellitus (T2DM), in addition to obesity, irregular menstrual cycle, hyperlipidemia, and cardiovascular diseases (Sachdeva, et al. ${ }^{[4]}$ ). Moreover, endometrial carcinoma (EC) is one of the metabolic long-term morbidity resulted from the syndrome (Rotterdam ESHRE/ASRM-Sponsored PCOS Consensus Workshop Group $\left.{ }^{[5]}\right)$. In winter of 2015, the American College of Endocrinology (ACE), American
Association of Clinical Endocrinologists Excess (AACE), and Androgen Excess and PCOS Society (AE-PCOS) advanced a new guideline in the management of PCOS. They have recommended that at least two of the following features should be present to diagnose PCOS (Goodman, et al. ${ }^{[6]}$ ) these were known as Rotterdam criteria. Chronic anovulation, androgen excesses (clinical or biologic), and polycystic appearance of the ovary on ultrasound (PCOM). Other causes of anovulatory infertility and hyper and roginemea should be excluded. Clinical findings, history, are important to reaching a diagnosis, in addition, assessment of free testosterone (FT) and (AMH) was recommended. It had been found that an elevated number of preantral and small antral follicles were reflected by the increase in anti-Müllerian hormone $(\mathrm{AMH})$, so it can replace ultrasound examination, but this still under experimental study. (AMH) can aid in the diagnosis of the syndrome and may be added to diagnostic criteria (Filippou and 
Homburg $\left.{ }^{[7]}\right)$. Various risk factors for diabetes are present in women with PCOS. These include insulin dysfunction, increased body mass index to more than 30 $\mathrm{kg} / \mathrm{m}^{2}$, and a family history of type 2 diabetes. Evidence of abnormal vascular function and dyslipidemia causes risk for cardiovascular disease (Tokmak, et al. ${ }^{[8]}$ ). The risk of ovarian cancer (OC) is increased in polycystic ovarian syndrome which is a form of hyperandrogenic state, which stimulates inactive androgen receptor (AR) and induces abnormal ovarian growth proliferation (Singh, et al. $\left.{ }^{[9]}\right)$. An androgenic compound used in the treatment of endometriosis increases the risks of ovarian cancer (OC) to more than three folds. This is regarded as evidence to support the androgen theory in ovarian cancer (Zhu, et al. $\left.{ }^{[10]}\right)$. Ovulationinducing drugs used in treating infertility in PCOS patients had an association with ovarian cancer (Balen, A. ${ }^{[11]}$ ). Infertility by itself can increase cancer risk to two folds, studies had been done on clomiphene citrate and other types of drugs used in treating anovulation concluded that their use more than six months in nulligravida women and still nulligravida increases the risk of borderline ovarian cancer, these studies dealing with infertility drugs still not reaching answer yet. In IVF procedures the increased risk of developing borderline ovarian tumors needs further studies. while sterilization, childbirth, and hysterectomy do not give protective effects against ovarian tumors (Momenimovahed, et al. ${ }^{[12]}$ ). Incessant ovulation theory explains that any condition which suppresses ovulation, which is the cause of ovarian epithelium damage, can give protection against cancer. So PCOS according to this theory, is regarded as protective against ovarian tumors (Fleming, et al. ${ }^{[13]}$ ). Overweight risk in women and PCOS patients can be explained as adipose tissue contains aromatase, which converts androstenedione to estrone and testosterone to estradiol (Balen, A. ${ }^{[11]}$ ), a situation where these women have both 
excessive androgens and estrogens which increase the risk for breast cancer (Gardener, et al. ${ }^{[14]}$ ). mitogenic effect of estrogen produced by an elevated level of the FSH and LH used in the treatment of infertility in the follicular phase explains the role of Gonadotropin in the occurrence of breast cancer (Schneider, et al. ${ }^{[15]}$ ). Endometrial cancer in women with PCOS has 2.7 times higher risk (Mujawar, et al. $\left.{ }^{[16]}\right)$ these include LH hypersecretion, chronic hyperinsulinemia, obesity, hyperandrogenism, and increased serum insulin-like growth factor [IGF]-I levels (Allahbadia and Merchant ${ }^{[17]}$ ). Estrogens unopposed by progesterone leading to endometrial hyperplasia over a period of years and causes increased risk for endometrial cancer. Furthermore, using combined oral contraceptive (coc) estrogen-progestin, as well as ART ovulation induction drugs have an association with endometrial cancer (Momenimovahed, et al. ${ }^{[12]}$; Mujawar, et al. ${ }^{[16]}$; Gadducci, et al. ${ }^{[18]}$ ). Endometrial biopsy and hysteroscopy have been recommended in these cases (Alsadi, B. $\left.{ }^{[19]}\right)$. As a result of these factors, screening by using CA125 tumor marker may be need.

\section{Cancer antigen 125 (CA125)}

Cancer antigen 125 (CA125) is a soluble protein, belongs to the mucin family; CA125 is composed of three different domains: N-terminal domain, tandemrepeat domains, and $\mathrm{C}$-terminal domains which are characterized by a short cytoplasmic tail. It is rich in the onine, serine, and proline. High in molecular weight (Mol. Wt.) about 3-5 million Da (Singh, et al. ${ }^{[9]}$ ). CA125 is expressed by normal bronchial epithelial cells and digestive tract, endometrial, ovarian, and corneal epithelium. This tumor marker is valuable in the antiviral, antibacterial role, and protection against injuries and toxins. Keeping epithelial surfaces moist and hydrated is needed for the proper functioning of ducts, and passageways (Felder, et al. $\left.{ }^{[20]}\right)$. CA125 concentration increased in physiological conditions such as ovulation, menstruation, and during 
pregnancy. In some benign conditions elevation of CA125 can be shown in endometriosis ovarian hyperstimulation syndrome (OHHS), ovarian cyst, ectopic pregnancy fibroid, and tubo-ovarian abscess (Sharma, S. ${ }^{[21]}$ ). Clinical implications of CA125 regarding malignancy can be classified into 4 groups: screening among the grouped population for early detection, diagnostic verification, prognosis, and prediction monitoring recurrence of cancer. CA125 is valuable for dealing with ovarian, endometrial, fallopian tubes cancers, as well as those cancers of the lung, breast, and gastrointestinal tract (pancreatic and colonic). Epididymis Protein 4 (HE4) can be used with CA125 to improve sensitivity and specificity of it (Chen, et al. ${ }^{[22]}$ ), other procedures of investigations can be combined with tumor markers e.g., a combination of Carbohydrate antigen CA125 with age by history and bimanual examination, or combining with ultrasonography in assessing pelvic mass (Aithal, et al. ${ }^{[23]}$ ). In detecting the recurrence of cancer by tumor marker, serial assessment of tumor marker is needed. Biochemical recurrence was seen when elevation in the concentration of tumor marker before any clinical or radiological evidence of the disease appear, additional radiological examination may be arranged to confirm the recurrence (Sharma, S. ${ }^{[21]}$; Aithal, et al. $\left.{ }^{[23]}\right)$.

\section{Materials and Methods}

This prospective cohort study had been done at the infertility clinic of the High Institute of Infertility Diagnosis and Assisted Reproductive Technologies at Al Nahrain University, Baghdad, Iraq in the period from July 2019 to October 2019. Ethical approval issued by the local Medical Ethical Committee at the university. Patients were informed about the study and written informed consent was obtained from each participant. The present study was done on forty PCOS women regardless of their marital state. 
Patients were grouped into three groups each one included twenty women:

\section{Group 1 (G1) were PCOS $\geq 25$ kg/m²}

\section{Group 2 (G2) were $\mathrm{PCOS}<25 \mathrm{~kg} / \mathrm{m}^{2}$}

\section{Group 3 (G3) were non-PCOS $<25 \mathrm{~kg} / \mathrm{m}^{2}$}

Physical assessment and biochemical investigations were done in the infertility clinic at the University. Weight and height were measured for every female to calculate their body mass index (BMI) according to the equation:

\section{BMI = Weight $(\mathrm{kg}) /$ Height $\left(\mathrm{m}^{2}\right)$}

\section{Inclusion criteria:}

Age (18-42) years, BMI $>18.5 \mathrm{~kg} / \mathrm{m}^{2}$ not pregnant at the time of assessment. PCOS patients had been chosen from patients who attended the institute and were diagnosed according to Rotterdam criteria (Lujan, et al. [24]), and healthy females who volunteer to join this study, no history of endocrine disease or endometriosis or an ovarian cyst.

\section{Exclusion criteria:}

Out of range of age <18-42> years. BMI $<18.5 \mathrm{~kg} / \mathrm{m}^{2}$ (underweight) pregnant females at the time of assessment, pelvic mass by U/S as cyst or fibroids, chronic diseases, hormonal disturbance as thyroid dysfunction, Cushing's syndrome hyperprolactinemia, hypogonadotropic hypogonadism, and congenital adrenal hypertrophy.

\section{Examinations:}

Physical and gynecological examination in addition to pelvic U/S for confirmation of PCOS diagnosis and exclusion of any mass. For all female's chemical investigation was done for detection of CA125 level and hormonal examination include LH, FSH, Free T. done by ELISA. Progesterone was given to induce bleeding in case of amenorrhea. The gynecological examination was done for all females included in the study. The body weight, height, body mass index (BMI) calculated by weight in kilogram divided by height in a square meter. Blood samples were 
collected from females, on the $2^{\text {nd }}-4^{\text {th }}$ day of their menstrual cycle. another sample was taken in the late follicular phase of the same cycle in CD 8-11. for measurement of CA125 Disposable syringes and plain plastic tubes were prepared and labeled. Each woman had a special forum written on it the name, date, serial number, cycle day. Venous blood samples of $5 \mathrm{ml}$ were taken by venous puncture. Kits used were CA125 Ag (ELISA) HUMAN Germany number of kit REF 52050 LOT 19001. Free Testosterone (ELISA) De medi tec Germany number of kit REF DE2924 LOT5050A. FSH HUMAN Germany number of kit REF 53020 LOT 17007. LH (ELISA) Monobind USA kit number LOT 6D1B8. Hormones and CA marker antigens were measured by EnzymeLinked Immunosorbent Assay (ELISA). Data collected were grouped and analyzed by using SPSS version 22 and Microsoft Office Excel 2010. Numeric variables were expressed as mean \pm standard deviation, range, numbers, and percentage (\%). Student t-test was used to compare independent two samples. Independent samples ANOVA (¥) was used to compare mean values between three groups, while the Chi-square test was used to study associations between any two categorical variables; however, Person's correlation coefficient (r) was used to study the correlation between two variables. The differences between values were considered statistically insignificant at the level of $(p>0.05)$ and significant at the level of $(\mathrm{p}<0.05)$ and highly significant at the level of $(\mathrm{p}<0.01)$.

\section{Results}

The demographic characteristics of the women enrolled in the current study were demonstrated in Table 1. There was no significant difference in mean age between $\mathrm{G} 1, \mathrm{G} 2$, and $\mathrm{G} 3),(\mathrm{P}=0.144)$ mean age in G1, G2, and G3 were (25.50 \pm 6.6$),(25.30 \pm 06.88),(28.05 \pm 6.07)$, respectively. As shown in Table 1 of G1 (obese PCOS) women were significantly higher $(\mathrm{P}<0.05)$ than the normal weight G2, G3. Mean \pm SD of BMI G1, G2, G3 
between BMI were $(34.41 \pm 5.80)$, (22.53 \pm 2.01$),(22.66 \pm 1.91)$, respectively. The highly significant difference in mean serum luteinizing hormone (LH) among study groups $(\mathrm{P}<0.001)$. The level was highest in PCOS obese women (G1) followed by PCOS normal weight (G2) women then control normal weight nonPCOS group (G3) with values of (10.24 \pm 5.68$),(9.10 \pm 3.42), \quad(5.49 \pm 1.60)$, respectively. Indeed, no significant difference between PCOS groups G1, G2, $\mathrm{P}>0.05$. There was no significant difference in mean serum folliclestimulating hormone (FSH) among study groups $\quad(\mathrm{P}=0.467), \quad$ being $\quad(7.11 \pm 1.88)$ $(7.25 \pm 1.72) \quad(6.77 \pm 1.48) \quad$ respectively. There was a highly significant difference in mean serum testosterone among study groups $(\mathrm{P}<0.001)$. The level was elevated compared to normal. It was highest in PCOS obese (G1) women followed by PCOS normal weight (G2) women than by control normal weight (G3) women. There was no significant difference in mean testosterone level between both PCOS groups $\quad(p>0.05)$,

$(1.52 \pm 0.69)$, (1.22 \pm 0.59$),(0.81 \pm 0.21)$, respectively as shown in Table 2. There was a highly significant difference in the mean LH/FSH ratio among study groups $(\mathrm{P}=0.001)$. The ratio was highest in PCOS obese G1 women followed by PCOS normal weight $\mathrm{G} 2$ women than by control normal weight G3 women. Indeed, there was no significant difference in mean LH/FSH level between both PCOS groups $(\mathrm{p}>0.05), \quad$ as follows

$(1.45 \pm 0.83)$ $(1.39 \pm 0.72)$ then G3 $(90.85 \pm 0.39)$ respectively. The LH/FSH ratio was elevated more than one in PCOS groups as shown in Table 2. There was a significant difference in mean serum CA125 among study groups $(\mathrm{P}=0.05) \mathrm{CA} 125$ level of women within the control normal weight group (G3) was the heights followed by PCOS normal weight (G2) women, then by PCOS obese (G1) women mean $(\mathrm{p}<0.05)$ being $\quad(15.40 \pm 2.53 \mathrm{U} / \mathrm{ml})$ $(15.38 \pm 4.16 \mathrm{U} / \mathrm{ml})(12.15 \pm 5.05)$. 
Table (1): Demographic characteristics of women enrolled in the present study

\begin{tabular}{|c|c|c|c|c|}
\hline Characteristic & $\begin{array}{c}\text { Control (G3) } \\
\text { normal weight } \\
n=20\end{array}$ & $\begin{array}{c}\operatorname{PCOS}(G 2) \\
\text { normal weight } \\
n=20\end{array}$ & $\begin{array}{c}\text { PCOS }(G 1) \\
\text { obese } \\
n=20\end{array}$ & $P$ \\
\hline \multicolumn{5}{|c|}{ Age (Years) } \\
\hline Mean $\pm S D$ & $\begin{array}{c}28.05 \pm 6.07 \\
\text { A }\end{array}$ & $\begin{array}{c}25.30 \pm 6.88 \\
A\end{array}$ & $\begin{array}{c}25.50 \pm 6.65 \\
\mathrm{~A}\end{array}$ & \multirow{2}{*}{$\begin{array}{l}0.144 \dagger \\
\quad \mathrm{NS}\end{array}$} \\
\hline Range & $19-38$ & $18-39$ & $18-41$ & \\
\hline$<35, n(\%)$ & $16(80.0 \%)$ & $18(90.0 \%)$ & $18(90.0 \%)$ & \multirow{2}{*}{$\begin{array}{c}0.667 ¥ \\
\text { NS }\end{array}$} \\
\hline$\geq 35, n(\%)$ & $4(20.0 \%)$ & $2(10.0 \%)$ & $2(10.0 \%)$ & \\
\hline \multicolumn{5}{|c|}{$B M I\left(k g / m^{2}\right)$} \\
\hline Mean $\pm S D$ & $\begin{array}{c}22.66 \pm 1.91 \\
B\end{array}$ & $\begin{array}{c}22.53 \pm 2.01 \\
B\end{array}$ & $\begin{array}{c}34.41 \pm 5.80 \\
\mathrm{~A}\end{array}$ & \multirow{2}{*}{$\begin{array}{c}<0.001 \dagger \\
\text { HS }\end{array}$} \\
\hline Range & $18.5-24.71$ & $19.28-24.9$ & $25.6-46.66$ & \\
\hline Normal, $n(\%)$ & $20(100.0 \%)$ & $20(100.0 \%)$ & $0(0.0 \%)$ & \multirow{3}{*}{$\begin{array}{c}<0.001 ¥ \\
\text { HS }\end{array}$} \\
\hline Overweight, $n(\%)$ & $0(0.0 \%)$ & $0(0.0 \%)$ & $5(25.0 \%)$ & \\
\hline Obese, $n(\%)$ & $0(0.0 \%)$ & $0(0.0 \%)$ & $15(75.0 \%)$ & \\
\hline
\end{tabular}

n: number of cases; SD: Standard deviation; BMI: Body mass index; $\uparrow$ : One-way ANOVA test; $¥$ : Chi-square test; NS: Not significant at P> 0.05; HS: Highly significant at $\mathrm{P} \leq 0.01$; Capital letters were used to indicate the level of significance following LSD multiple comparison test or 2 groups Chi-square test so that similar letters indicate no significant difference, while, different letters indicate significant difference; letter (A) takes the highest value followed by (B). 
Table (2): Serum hormonal levels and CA125 of women with PCOS and control women

\begin{tabular}{|c|c|c|c|c|}
\hline Characteristic & $\begin{array}{c}\text { Control G3 } \\
\text { normal weight } \\
n=20\end{array}$ & $\begin{array}{c}\text { PCOS G2 } \\
\text { normal weight } \\
n=20\end{array}$ & $\begin{array}{l}\text { PCOS G1 } \\
\text { obese } \\
n=20\end{array}$ & $\boldsymbol{P}$ \\
\hline \multicolumn{5}{|c|}{ Luteinizing hormone (LH) } \\
\hline Mean $\pm S D$ & $\begin{array}{c}5.49 \pm 1.60 \\
B\end{array}$ & $\begin{array}{c}9.10 \pm 3.42 \\
A\end{array}$ & $\begin{array}{c}10.24 \pm 5.68 \\
A\end{array}$ & \multirow{2}{*}{$\begin{array}{c}<0.001+ \\
\mathrm{HS}\end{array}$} \\
\hline Range & $3.14-8.46$ & $2.5-15.7$ & $2.64-26.22$ & \\
\hline \multicolumn{5}{|c|}{ Follicle-stimulating hormone (FSH) } \\
\hline Mean $\pm S D$ & $\begin{array}{c}6.77 \pm 1.48 \\
A\end{array}$ & $\begin{array}{c}7.11 \pm 1.88 \\
A\end{array}$ & $\begin{array}{c}7.25 \pm 1.72 \\
A\end{array}$ & \multirow{2}{*}{$\begin{array}{c}0.467+ \\
\text { NS }\end{array}$} \\
\hline Range & $3.25-9.26$ & $3.71-9.27$ & $2.90-9.64$ & \\
\hline \multicolumn{5}{|c|}{ Testosterone } \\
\hline Mean $\pm S D$ & $\begin{array}{c}0.81 \pm 0.21 \\
B\end{array}$ & $\begin{array}{c}1.22 \pm 0.59 \\
A\end{array}$ & $\begin{array}{c}1.52 \pm 0.59 \\
A\end{array}$ & \multirow{2}{*}{$\begin{array}{c}<0.001+ \\
\text { HS }\end{array}$} \\
\hline Range & $0.62-1.30$ & $0.61-2.41$ & $0.65-2.9$ & \\
\hline \multicolumn{5}{|c|}{ LH/FSH ratio } \\
\hline Mean $\pm S D$ & $\begin{array}{c}0.85 \pm 0.39 \\
B\end{array}$ & $\begin{array}{c}1.39 \pm 0.72 \\
A\end{array}$ & $\begin{array}{c}1.45 \pm 0.83 \\
A\end{array}$ & \multirow{2}{*}{$\begin{array}{c}0.001+ \\
\mathrm{HS}\end{array}$} \\
\hline Range & $0.53-2.45$ & $0.27-3.18$ & $0.44-3.69$ & \\
\hline \multicolumn{5}{|c|}{ Serum Ca-125 (U/ml) } \\
\hline Mean $\pm S D$ & $\begin{array}{c}15.40 \pm 2.53 \\
\mathrm{~A}\end{array}$ & $\begin{array}{c}15.38 \pm 4.16 \\
\mathrm{~A}\end{array}$ & $\begin{array}{c}12.15 \pm 5.05 \\
B\end{array}$ & \multirow{2}{*}{$\begin{array}{c}0.001 \dagger \\
\mathrm{HS}\end{array}$} \\
\hline Range & $7.08-18.07$ & $7.48-22.68$ & $5.38-23.06$ & \\
\hline
\end{tabular}

n: number of cases; SD: Standard deviation; $\uparrow$ : One-way ANOVA test; NS: Not significant at P>

0.05; HS: Highly significant at $\mathrm{P} \leq 0.01$; Capital letters were used to indicate the level of significance following LSD multiple comparison test so that similar letters indicate no significant difference, while, different letters indicate significant difference; letter (A) takes the highest value followed by (B), (C) and (D) 
Table (3): Correlations of serum CA125 to other variables in women enrolled in the present study

\begin{tabular}{|c|c|c|c|c|c|c|}
\hline \multirow{2}{*}{ Characteristic } & \multicolumn{2}{|c|}{$\begin{array}{c}\text { Control C3 } \\
\text { normal weight } \\
\boldsymbol{n = 2 0}\end{array}$} & \multicolumn{2}{c|}{$\begin{array}{c}\text { PCOS C2 } \\
\text { normal weight } \\
\boldsymbol{n}=\mathbf{2 0}\end{array}$} & \multicolumn{2}{c|}{$\begin{array}{c}\text { PCOS G1 } \\
\text { obese } \\
\boldsymbol{n}=\mathbf{2 0}\end{array}$} \\
\cline { 2 - 7 } & $\boldsymbol{r}$ & $\boldsymbol{P}$ & $\boldsymbol{r}$ & $\boldsymbol{P}$ & $\boldsymbol{r}$ & $\boldsymbol{P}$ \\
\hline Age & 0.039 & 0.870 & 0.728 & $<0.05^{*}$ & -0.113 & 0.635 \\
\hline L.H & -0.151 & 0.525 & 0.185 & 0.436 & 0.146 & 0.539 \\
\hline F.S.H & -0.057 & 0.810 & -0.164 & 0.489 & 0.213 & 0.368 \\
\hline Testosterone & 0.498 & $0.026^{*}$ & 0.151 & 0.525 & -0.092 & 0.701 \\
\hline
\end{tabular}

:*Significant at $\mathrm{P} \leq 0.05$; **: Significant at $\mathrm{P} \leq 0.01$; ---: Correlation cannot be calculated because one variable is constant

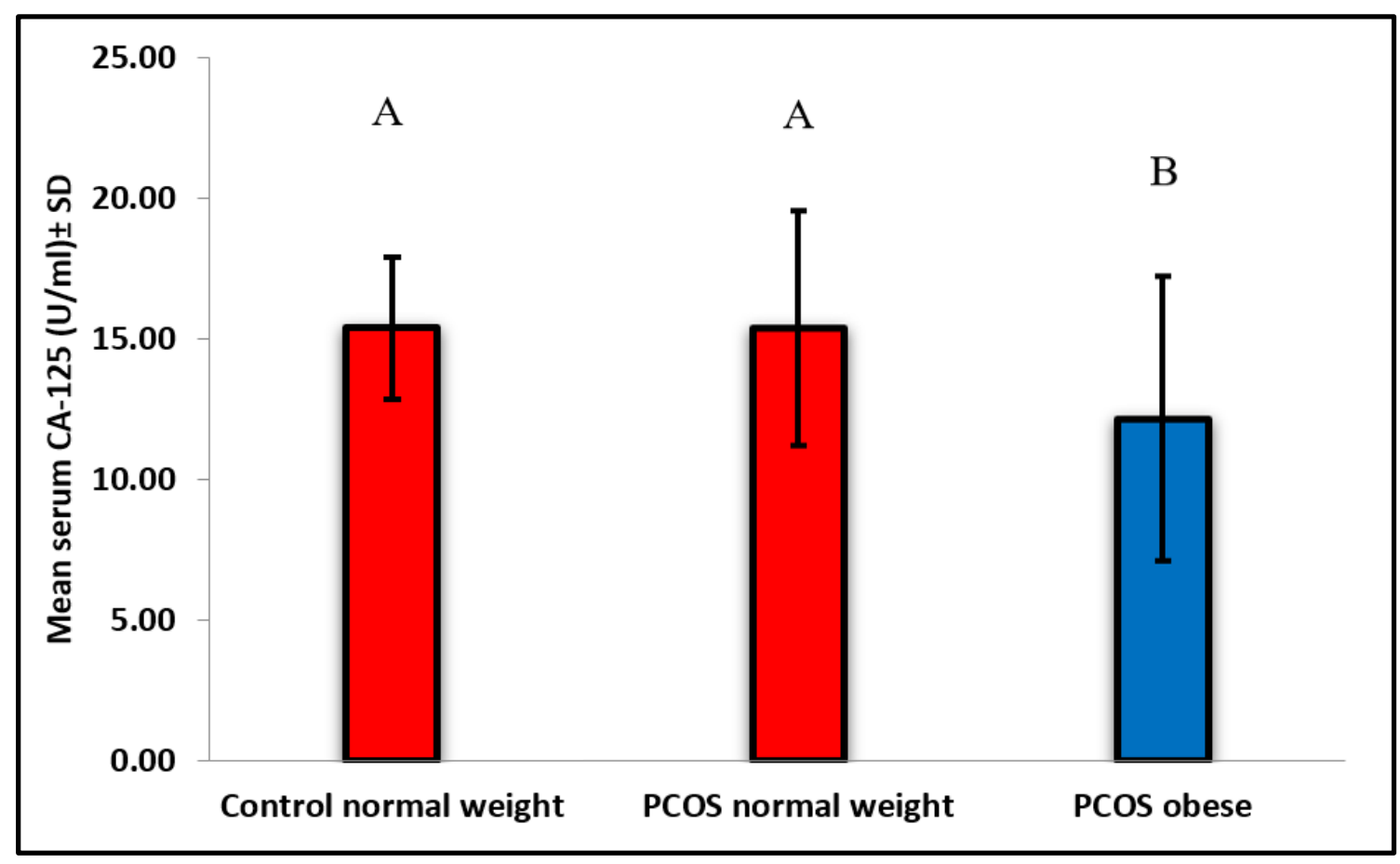

Figure (1): Serum CA125 in PCOS women and control women 
No significant difference in CA125 level between normal-weight PCOS G2 and normal weight $\mathrm{G} 3$ non-PCOS $(\mathrm{P}>0.05)$ as shown in Table (2) and Figure (1) $(15.40 \pm 2.53 \mathrm{U} / \mathrm{ml}) \quad(15.38 \pm 4.16 \mathrm{U} / \mathrm{ml})$. Indeed, there was a significant negative correlation between BMI and serum CA125 in women enrolled in the current study $(r=-0.750 ; p<0.05)$. This correlation is explained as follows: the higher the body mass index is, the lower the serum CA125 level is. Concerning CA125 in PCOS groups, there was an insignificant weak relation with testosterone and significant relation with testosterone in G3.

\section{Discussion}

As far as our knowledge small number of studies done on CA125 in non-cancerous women, moreover, there is a small number of studies that deal with PCOS and CA125 tumor markers. In this study no difference in mean and SD of women according to age which removes any variations that may affect the results of the measured biochemical parameter. The significant difference in BMI among the study groups to study the relation of CA125 with PCOS according to BMI, G1 significantly difference from $\mathrm{G} 2$, but there is no significant difference between G2 and G3 (non-PCOS of normal weight). Excess adipocyte in obese women with PCOS results in worsening of the reproductive and metabolic outcomes. Obese women with PCOS compared to women with normal weight PCOS have more occurrence of glucose intolerance and type 2 diabetes mellitus, obstructive sleep apnea, as well as a greater risk of cardiovascular disease and metabolic syndrome. In addition, obese patients have a greater risk of endometrial cancer (Alsadi, B. ${ }^{[19]}$ ). There was a highly significant difference in mean serum luteinizing hormone (LH) among study groups $(\mathrm{p}<0.001)$. The level was highest in PCOS obese group (G1) followed by PCOS normal weight group (G2) the control normal group (G3). Indeed, there was no significant difference in mean LH 
level between both PCOS groups ( $p>0.05)$, Table (2) The result of the present study shows that LH in PCOS groups compared to (healthy non-PCOS) was greater level or normal, so it copes with many previous studies such as a study done in 2016 by (De Leo, et al. ${ }^{[25]}$ ). A weak and non-significant correlation between LH and CA125 was seen among study groups as shown in Table (3). There was no significant difference among groups in Follicular Stimulating Hormone (FSH) $(\mathrm{p}=0.4670 . \mathrm{FSH}$ was within the normal range (2.9-10.88) and many studies show that FSH was normal or low in PCOS (Park and Chun ${ }^{[1]}$ ). FSH may be at a normal level but not functioning because follicular development is arrested by increased $\mathrm{AMH}$ which was blamed in anovulation by inhibiting FSH action (Filippou, et al. ${ }^{[7]}$ ) in this research, had been found that FSH was of a normal level. Weak and insignificant relation seen between CA125 and FSH among study groups and CA125, as shown in Table 3. The LH/FSH was more than one in PCOS groups being a highly significant difference between PCOS groups and non-PCOS, Hsu mentioned that an LH/FSH ratio higher than 1 gives the best combination of sensitivity and specificity for the diagnosis of PCOS (Hsu, M. $\left.{ }^{[26]}\right)$. There was an inverse correlation between BMI and CA125 level. which means that when the BMI was high the CA125 was low. In the current study no significant difference between G2 and G3 in the concentration of CA125, indeed there is no significant difference in BMI between the two groups that mean even there were hormonal disturbance and highly significant difference in hormones between (PCOS) and healthy (non-PCOS) but the level of CA125 related to BMI and no significant difference in the level of CA125 so hormonal disturbance prevent ovulation and decrease the damage or injury to the ovarian epithelium (Fleming, et al. ${ }^{[13]}$ ). So, serum CA125 is not elevated by PCOS. This result was agreed with the result of (Rzaij, Z. $\left.{ }^{[27]}\right)$ study done in 2012 in Iraq on Iraqi PCOS women in 
premenopausal age where the concentration of CA125 marker was within normal there was no significant difference between the normal sample and PCOS sample she explained that by as: even there was a hormonal disturbance in PCOS women, still, there were no stimulate on to the serosal surface of the ovaries which is the main causes for elevation in CA125 (Rzaij, Z. ${ }^{[27]}$ ). In contrast to the current study, Mujawaretal found that there were significantly higher $(p<0.001)$ serum CA125 levels in PCOS patients than in the control group (Momenimovahed, et al. ${ }^{[12]}$ ). In this study there was a negative relation between CA125 and BMI in a noncancerous patient this is because of the dilution effect of large plasma volume in obese. This concluded a decrease in efficacy of CA125 in obese women. In his study done on, Korean women aged more than 40 years, Kim, in the period between March 2007 and March 2013 large study on women and men for screening ca ovary and ca prostate by tumor markers CA125 for women and Prostate Specific Antigen (PSA) for Prostate cancer, in men as it is one of the most common malignant tumors among men, this cancer closely associated with obesity and, thus far, several studies have suggested the association between obesity and aggressive pathological behavior of prostatic cancer never the less they were associated with a decrease in the serum level of tumor marker prostate-specific antigen (PSA). This was because of the dilution effect of plasma volume associated with obesity (Kim, et al. ${ }^{[28]}$ ). In obesity plasma volume increases and in non-cancerous conditions there is a fixed amount of tumor marker dissolved in a larger volume of plasma, so dilute the concentration of the maker (Kim, et al. $\left.{ }^{[281}\right)$. Another study on 2560 postmenopausal women level of CA125 was related negatively with BMI in nonovarian cancer women (Johnson, et al. $\left.{ }^{[29]}\right)$. 


\section{Conclusions}

PCOS does not cause an increase in CA125. The negative relation between CA125 concentration and weight, when BMI high the concentration of CA125 decreases. The tumor marker CA125 efficacy is decreased in obese women.

\section{Acknowledgment}

We would like to acknowledge Al Nahrain University, Baghdad, Iraq.

\section{Funding}

This work received no funding.

\section{Author Contribution}

Hasan, HA performed the study, examined and reviewed results, and manuscript writing with help and supervision of Selman MO, and Jwad MA.

\section{Conflict of Interest}

The author declares no conflict of interest.

\section{Ethical Clearance}

The study was approved by the Ethical Approval Committee.

\section{References}

[1]Park C-H, Chun S. Association between serum gonadotropin level and insulin resistance-related parameters in Korean women with polycystic ovary syndrome. Obstet Gynecol Sci. 2016;59(6):498. [Online Article Link]

[2] Kabel A. Polycystic Ovarian Syndrome: Insights into Pathogenesis, Diagnosis, Pathog Pcos. 2016;1(1):15. [Online Article Link]

[3] Selman MO, Al-hassani WR, Al-wasiti E, Mahdi KT, Suhail AM. Role of IGF 1, VEGF, Vit. D3 and Vit. B12 in high AMH level in Iraqi Infertile women as criteria to prepare them for IVF. 2019;7(7):237-40 .[Online Article Link]

[4] Sachdeva G, Gainder S, Suri V, Sachdeva N, Chopra S. Comparison of the different pcos phenotypes based on clinical metabolic, and hormonal profile, and their response to clomiphene. Indian $\mathrm{J}$ Endocrinol Metab. 2019;23(3):326 .[Online Article Link]

[5] Rotterdam ESHRE/ASRM-Sponsored PCOS Consensus Workshop Group. Revised 2003 consensus on diagnostic criteria and long-term health risks related to polycystic ovary syndrome. Vol. 81, Fertility and Sterility. 2004. p. 19-25. [Online Article Link]

[6] Goodman NF, Cobin RH, Futterweit W, Glueck JS, Legro RS, Carmina E. 
American Association of Clinical Endocrinologists, American College of Endocrinology, and Androgen Excess and PCOS Society. American Association of Clinical Endocrinologists, American College of Endocrinology, and Androgen Excess and Pcos Society Disease State Clinical Review: Guide to the Best Practices in the Evaluation and Treatment of Polycystic Ovary Syndrome - Part 1, Endocrine Practice. 2015:21(11), 1291-1300. [Online Article Link]

[7]Filippou P, Homburg R. Is foetal hyperexposure to androgens a cause of PCOS ? Human Reproduction Update. 2017;23(4):421-32 . [Online Article Link]

[8] Tokmak A, Kokanali MK, Guzel AI, Kara A, Topcu HO, Cavkaytar S. Polycystic ovary syndrome and risk of endometrial cancer: A mini-review, Asian Pacific Journal of Cancer Prevention, 2014;15(17):7011-7014. [Online Article Link]

[9] Singh AP, Senapati S, Ponnusamy, MP, Jain M, Lele SM, Davis JS, Remmenga S, Batra, SK. Clinical potential of mucins in diagnosis, prognosis, and therapy of ovarian cancer. Lancet Oncol. 2008;9(11):1076-85. [Online Article Link]

[10] Zhu H, Zhu X, Zheng L, Hu X, Sun, $\mathrm{L}$, Zhu $\mathrm{X}$. The role of the androgen receptor in ovarian cancer carcinogenesis and its clinicalimplications. Oncotarget, 2017;8:29395-29405. [Online Article Link]

[11] Balen A. Polycystic ovary syndrome and cancer, Human Reproduction Update, 2001;7(6):522525. [Online Article Link]

[12] Momenimovahed Z, Taheri S, Tiznobaik A, Salehiniya H. Do the Fertility Drugs Increase the Risk of Cancer? A Review Study. Front Endocrinol (Lausanne). 2019 May 24;10:313. [Online Article Link]

[13] Fleming JS, Beaugie CR, Haviv I, Chenevix-Trench G, Tan OL. Incessant ovulation, inflammation and epithelial ovarian carcinogenesis: Revisiting old hypotheses Jean. Molecular and Cellular Endocrinology, 2006 Mar 9;247(12):4-21. [Online Article Link]

[14] Gardener DK, Ariel W, Haowles CM, Shoham Z (eds.). Textbook of Assisted Reproductive Techniques. $5^{\text {th }}$ edition. CRC Press; 2018. [Online Article Link]

[15] Schneider J, Martín-Gutiérrez S, Tresguerres JA, García-Velasco JA. Circulating estradiol defines the tumor phenotype in menopausal breast cancer patients. Maturitas. 2009;64(1):43-5. [Online Article Link]

[16] Mujawar SA, Kurude VN, Gaikwad HA, Patil VW. Utility of ovarian 
tumour marker cancer antigen-125 and endocrine hormonal status in polycystic ovary syndrome. J Clin Diagnostic Res. 2018 Oct 1;12(10):BC01-3. [Online Article Link]

[17] Allahbadia GN, Merchant R. Polycystic ovary syndrome and impact on health. Middle East Fertility Society Journal. 2011;16(1):19-37. [Online Article Link]

[18] Gadducci A, Gargini A, Palla E, Fanucchi A, Genazzani AR. Polycystic ovary syndrome and gynecological cancers: Is there a link? Gynecological Endocrinology. $\quad 2005 ; 20(4): 200-8$. [Online Article Link]

[19] Alsadi B. Polycystic Ovary Syndrome, Pathophysiology, and Reproductive Health Implications, In Pathophysiology, Gaze DC (ed.), Intech, 2018. [Online Article Link]

[20] Felder M, Kapur A, GonzalezBosquet J, Horibata S, Heintz J, Albrecht R, et al. MUC16 (CA125): Tumor biomarker to cancer therapy, a work in progress. Mol Cancer. 2014;13:129. [Online Article Link]

[21] Sharma S. Tumor markers in clinical practice: General principles and guidelnes. Indian $\mathrm{J}$ Med Paediatr Oncol. 2009;30(1):1-8. [Online Article Link]

[22] Chen F, Shen J, Wang J, Cai P, Huang Y. Clinical analysis of four serum tumor markers in 458 patients with ovarian tumors: Diagnostic value of the combined use of HE4, CA125, CA19-9, and CEA in ovarian tumors. Cancer Manag Res. 2018 May 22;10:1313-8. [Online Article Link]

[23] Aithal A, Rauth S, Kshirsagar P, Shah A, Lakshmanan I, Junker WM, et al. MUC16 as a novel target for cancer therapy. Expert Opinion on Therapeutic Targets. Taylor and Francis Ltd; 2018;22:675-86. [Online Article Link]

[24] Lujan ME, Chizen DR, Pierson RA. Diagnostic Criteria for Polycystic Ovary Syndrome: Pitfalls and Controversies. Journal of Obstetrics and Gynaecology Canada; 2008;30(8):671-679. [Online Article Link]

[25] De Leo V, Musacchio MC, Cappelli V, Massaro MG, Morgante G, Petraglia F. Genetic, hormonal and metabolic aspects of PCOS: an update Reproductive Biology and Endocrinology. BioMed Central Ltd. 2016;14:38. [Online Article Link]

[26] Hsu MI. Clinical characteristics in Taiwanese women with polycystic ovary syndrome. Clinical and experimental reproductive medicine. 2015:42(3):86-93. [Online Article Link]

[27] Rzaij ZF, AL-Ani NKH. Evaluation of Cancer Antigens (CA125\&CA153-) in some Iraqi women with polycystic ovarian 
syndrome. Iraqi $\mathbf{J}$ Embryos Infertil. Res. 2013;3(6):11-4. [Online Article Link]

[28] Kim JH, Park BR, Yang WJ. Dilution effect of serum CA125 a19-9 over a cutoff value, according to obesity, Int J Biol Markers. 2015 Feb 24;30(1):e122-6. [Online Article Link] [29] Johnson CC, Kessel B, Riley TL, Ragard LR, Williams CR, Xu JL, Buys SS, Prostate, Lung, Colorectal and Ovarian Cancer Project Team. The epidemiology of CA-125 in women without evidence of ovarian cancer in the Prostate, Lung, Colorectal and Ovarian Cancer (PLCO) Screening Trial. Gynecologic-oncology. 2008:110(3),383-9. [Online Article Link]

\section{Authors Biographies}

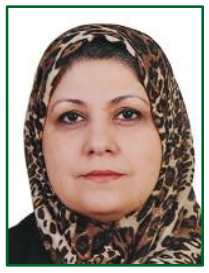

\section{Dr. Hanan Abdulmaged Hasan}

She is a General Practioner with a special interest in Dermatology. She graduated from The College of Medicine, Baghdad University in Iraq in 1987. She worked in Baghdad hospitals and medical centers then she retired for medical reasons. She has obtained a Master's degree in Applied Embryology in 2020.

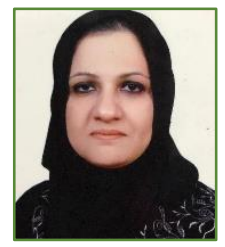

\section{Dr. Mufeda Ali Jwad}

She received her MBChB. From the College of Medicine at the University of Baghdad in 1996. Her M.Sc. in Applied Embryology and her Ph.D. in Infertility and Clinical Reproduction were from the High Institute of Infertility Diagnosis and Assisted

Reproductive Technologies, Al Nahrain University in 2007 and 2018 respectively. She worked as a rotator in the Baghdad health department from 1996-1999. She worked in Gyn. \& Obs. in Alsamawa general hospital and Babylon hospital from 2000-2003. She worked at the Babylon University, College of Medicine, anatomy and embryology department from 2003-2004. She has been working as a specialist physician and a consultant clinic at the High Institute of Infertility Diagnosis and Assisted Reproductive Technologies, Al Nahrain University from 2008-2015. Currently, she is an assistant professor and specialist in infertility and clinical reproduction. She is the head of the clinical reproductive physiology department from 2019 till now. She has more than 25 published articles in national and international journals.

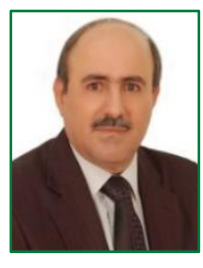
has articles both local and international. He supervised many M.Sc. and Ph.D. students

\section{How to cite:}

Hasan HA, Selman MO, Jwad MA. Effect of body mass index on serum CA125 level in females with PCOS; Iraqi Journal of Embryos and Infertility Researches (IJEIR), (2020); 10(1): 101-118. Doi: http://doi.org/10.28969/IJEIR.v10.i1.r7

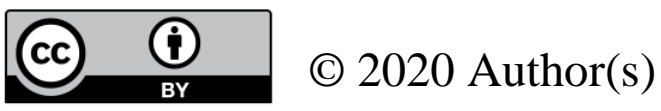

This article is licensed under a Creative Commons Attribution 4.0 International License, which permits use, sharing, adaptation, distribution and reproduction in any medium or format, as long as you give appropriate credit to the original author(s) and the source, provide a link to the Creative Commons license, and indicate if changes were made. http://creativecommons.org/licenses/by/4.0/. 